\title{
Intra-Patient Supine-Prone Colon Registration in CT Colonography Using Shape Spectrum
}

\author{
Zhaoqiang Lai ${ }^{1}$, Jiaxi $\mathrm{Hu}^{1}$, Chang $\mathrm{Liu}^{1}$, Vahid Taimouri ${ }^{1}$, Darshan $\mathrm{Pai}^{1}$, \\ Jiong $\mathrm{Zhu}^{2}$, Jianrong $\mathrm{Xu}^{2}$, and Jing $\mathrm{Hua}^{1}$ \\ 1 Computer Science, Wayne State University, Detroit, MI 48202, USA \\ 2 Radiology, Renji Hospital, Shanghai JiaoTong University, 200127, China \\ jinghua@wayne.edu
}

\begin{abstract}
CT colonography (CTC) is a minimally invasive screening technique for colorectal polyps and colon cancer. Since electronic colon cleansing (ECC) cannot completely remove the presence of pseudo-polyps, most CTC protocols acquire both prone and supine images to improve the visualization of the lumen wall and to reduce false positives. Comparisons between the prone and supine images can be facilitated by computerized registration between the scans. In this paper, we develop a fully automatic method for registering colon surfaces extracted from prone and supine images. The algorithm uses shape spectrum to extract the shape characteristics which are employed as the surface signature to find the correspondent regions between the prone and supine lumen surfaces. Our experimental results demonstrate an accuracy of $12.6 \pm 4.20 \mathrm{~mm}$ over 20 datasets. It also shows excellent potential in reducing the false positive when it is used to determine polyps through correspondences between prone and supine images.
\end{abstract}

Keywords: Colon Registration, Shape Spectrum, CT Colonography, Virtual Colonoscopy.

\section{Introduction}

Computed tomography colonography (CTC) has received increasing attention as a minimally invasive method for the examination of the colon 1]. CTC has shown promising results in the detection of clinically significant polyps. Using the advanced image technique, doctors can look for polyps throughout the entire colon via fly-through in a virtual colon model which is constructed from patient's abdominal images. In the last decade, many computer aided detection and diagnosis systems have been proposed and actively studied to improve the performance and reliability. In order to better differentiate polyps from pseudopolyps, and to better view the lumen surface in the presence of fluid, it is common practice to obtain two CT scans of the patient, one with the patient in the prone position and one in the supine. Based on this setup, the registration between the supine and prone colons is required.

Although many methods have been developed for shape registration [2]3], relatively fewer methods have been presented for registration of the supineprone colons. Li et al. 4] have developed methods to map candidate polyps 
between the supine and prone colons using their colon wall positions relative to the colon centerlines. This kind of method only takes into account the local extrema located on the inferior/superior axis which is not reliable due to colon shifts obliquely when the patient changes position. In such a case, some local extrema may not be considered. Nain et al. 5] presented a similar approach for aligning data along the centerlines used dynamic programming. Suh and Wyatt 6] used a piece-wise centerline matching algorithm and an interpolation and extrapolation method of deformation field for deformable registration. However, the deformable model requires the good initial alignment of the two colons and strict constraints. Otherwise, it might create artifacts which, in the worst case, result in pseudo-polyps. Besides the methods based on the centerlines, Näppi et al. 7] developed a directional region growing method for reducing false positive (FP) based on correspondence between the supine and prone datasets.

In this work, we developed a novel method for registration between the supine and prone datasets and reduced the FP based on their correspondence. Without matching the centerlines, we employed shape spectrum to extract the shape characteristics as the signature to find the correspondent regions between the prone and supine lumen surface. The method is simple yet efficient and accurate. Our contributions in this paper can be summarized as follows:

- A novel surface registration method based on shape spectrum is invented and applied to colon surface registration.

- We have applied the algorithm to the real CTC datasets and the experiments demonstrate the excellent accuracy of our registration results.

- We integrate the registration component into the virtual colonoscopy (VC) software for FP reduction, which shows that it is an excellent tool aiding polyp diagnosis in CTC.

\section{Methods}

Firstly, we discuss how to calculate the shape spectrum on the colon lumen surface to extract the shape characteristics which can be used as the signature for registration purpose. Next we detail how to apply the shape spectrum to the colon registration problem.

\subsection{Laplacian Shape Spectrum}

For a manifold $\mathcal{M}$, let $\Delta$ denote its Laplace-Beltrami differential operator 8 . Consider the Laplacian eigenvalue equation

$$
\Delta \phi=-\lambda \phi,
$$

where $\lambda$ is a real scalar which is called an eigenvalue of $\Delta$ and the $\phi$ is called an eigenvector corresponding to $\lambda$. The spectrum is defined to be the eigenvalues arranged increasingly as

$$
\lambda_{0}=0<\lambda_{1}<\lambda_{2}<\ldots<\lambda_{i}<\ldots<+\infty .
$$


The Laplace-Beltrami operator is Hermitian, so the eigenvectors corresponding to its different eigenvalues are orthogonal: $<\phi_{i}, \phi_{j}>=\int_{\mathcal{M}} \phi_{i} \phi_{j}=0$, where $i \neq j$.

Eq. 1 can be solved by the finite element method 9]. For colon surfaces, which are discrete triangle meshes, a discrete Laplace-Beltrami operator $K$ can be applied on it:

$$
K\left(p_{i}\right)=\frac{1}{2 A_{i}} \sum_{p_{j} \in N_{1}\left(p_{i}\right)}\left(\cot \alpha_{i j}+\cot \beta_{i j}\right)\left(p_{i}-p_{j}\right),
$$

where $N_{1}\left(p_{i}\right)$ includes all the vertices, $p_{j}$, belonging to one ring neighborhood of a vertex, $p_{i} . \alpha_{i j}$ and $\beta_{i j}$ are the two angles opposite to the edge in the two triangles sharing the edge $i, j$, and $A_{i}$ is the Voronoi region area of $p_{i}$.

For the whole vertices of a triangle mesh, a Laplace-Beltrami matrix can be constructed as:

$$
L_{i j}= \begin{cases}-\frac{\cot \alpha_{i j}+\cot \beta_{i j}}{2 A_{i}} & \text { if } \mathrm{i}, \mathrm{j} \text { are adjacent } \\ \sum_{k \in N_{1}\left(p_{i}\right)} \frac{\cot \alpha_{i k}+\cot \beta_{i k}}{2 A_{i}} & \text { if } \mathrm{i}=\mathrm{j} \\ 0 & \text { otherwise }\end{cases}
$$

where $\alpha_{i j}, \beta_{i j}$, and $A_{i}$ are the same as in Eq. 3 for certain $i$ and $j$. Then, Eq. 1 turns into the following eigenvalue problem:

$$
L \vec{v}=\lambda \vec{v}
$$

where $\vec{v}$ is an $n$-dimensional vector. Each entry of $\vec{v}$ represents the function value at one of $n$ vertices on the mesh. The equation above can be represented as a generalized eigenvalue problem which is much easier to solve numerically by constructing a sparse matrix $M$ and a diagonal matrix $S$ such that

$$
M_{i j}= \begin{cases}-\frac{\cot \alpha_{i j}+\cot \beta_{i j}}{2} & \text { if } \mathrm{i}, \mathrm{j} \text { are adjacent } \\ \sum_{k \in N_{1}\left(p_{i}\right) \frac{\cot \alpha_{i k}+\cot \beta_{i k}}{2}} & \text { if } \mathrm{i}=\mathrm{j} \\ 0 & \text { otherwise }\end{cases}
$$

and $S_{i i}=A_{i}$. Thus, the matrix $L$ is decomposed as $L=S^{-1} M$ and the generalized eigenvalue problem is presented as:

$$
M \vec{v}=\lambda S \vec{v}
$$

As defined above, $M$ is symmetric and $S$ is symmetric positive-definite.

The second eigenvector of the Laplacian is called the Fiedler vector and has interesting properties, making it a good permutation vector for numerical computations [10]. Fig. 1 (a) and (b) show that it naturally follows the shape of the colon mesh. In other words, the Fiedler vector defines a (1-dimensional) embedding of the surface mesh. We are trying to use the embedding for the registration of the colon surfaces. In next section we will detail how to do the registration. 

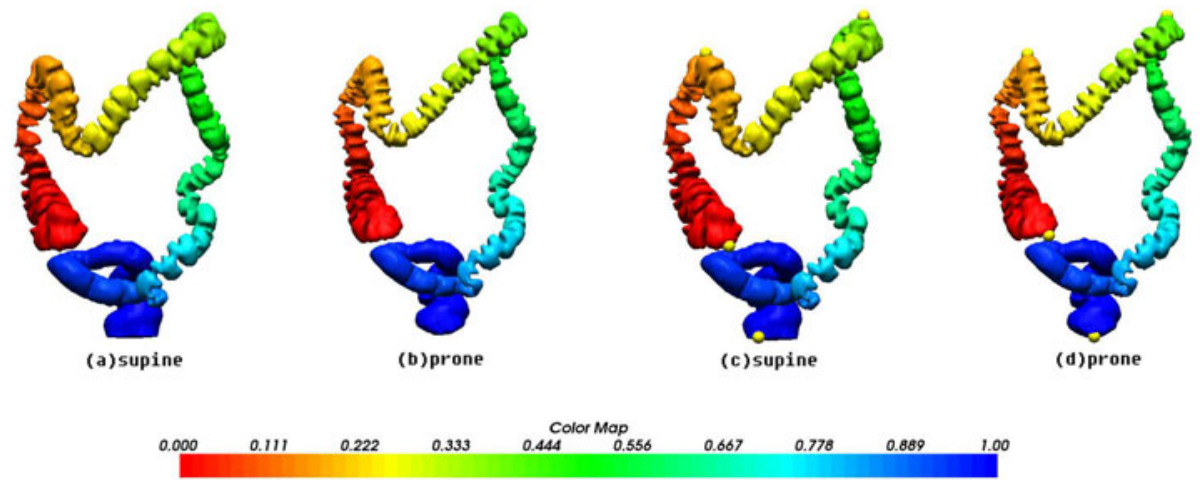

Fig. 1. Illustration of Fiedler vector embedding and landmarks detection. The Fiedler vector gives a natural ordering of the vertex of the colon meshes in (a) and (b). The vector value has been normalized and color map. (c) and (d) show the reliable automatic detected landmarks which are rendering in yellow balls.

\subsection{Registration Using Shape Spectrum}

First of all, after we obtained the colon surface mesh, we calculated the shape spectrum of the colon mesh. By constructing matrix $M$ and $S$ in Section 2.1, we solved the Eq. 7 to get the eigenvalues and eigenvectors.

To perform the registration efficiently, we first detect reliable anatomical landmarks. Based on the knowledge of colon anatomy, we expect that the mobility of the colon is at its smallest in these landmark regions. Four landmarks are established here: cecum, hepatic flexure, splenic flexure and anus. By using the normalized Fiedler vector value $\left(F_{v}\right)$, the cecum and anus are detected by determination of the smallest and largest $F_{v}$. This is done for both supine and prone datasets. For hepatic flexure and splenic flexure, we first process the supine dataset, and then deal with the prone one. For supine dataset, the hepatic flexure is found by detecting the local maximum z-coordinate whose $F_{v}$ is near 0 and the splenic flexure is found by detecting the local maximum z-coordinate whose $F_{v}$ is near 1 . For prone dataset, we set the neighborhood using the $F_{v}$ of hepatic flexure in supine as $\left[F_{v}-\varepsilon, F_{v}+\varepsilon\right]$. At this interval in prone dataset, we detect the local maximum z-coordinate as the hepatic flexure in prone. The splenic flexure in prone is detected with the same strategy. Fig. 1 (c) and (d) show the landmarks both in supine and prone datasets. After this procedure, we have landmarks in sequences: $L_{1}^{S}, L_{2}^{S}, L_{3}^{S}, L_{4}^{S}$ represent the cecum, hepatic flexure, splenic flexure and anus, respectively, in supine dataset and $L_{1}^{P}, L_{2}^{P}, L_{3}^{P}$, $L_{4}^{P}$ in prone dataset.

With the landmarks, we register the colon surface using piecewise registration. For each segment in supine between $L_{i}^{S}$ and $L_{i+1}^{S}$, we will map it to the segment in prone between $L_{i}^{P}$ and $L_{i+1}^{P}, i=1,2,3$. Let

$$
F_{v}^{S}\left(L_{i}^{S}\right), F_{v}^{S}\left(L_{i+1}^{S}\right) \text { and } F_{v}^{P}\left(L_{i}^{P}\right), F_{v}^{P}\left(L_{i+1}^{P}\right)
$$


represent the Fiedler vector value at the supine and prone location of $L_{i}^{S}, L_{i+1}^{S}$ and $L_{i}^{P}, L_{i+1}^{P}$, respectively. Then, for an examined location $L_{e}^{S}$ in supine between $L_{i}^{S}$ and $L_{i+1}^{S}$, the corresponding location $L_{e}^{P}$ in prone should have this relation of the $F_{v}$ :

$$
\frac{F_{v}^{S}\left(L_{e}^{S}\right)-F_{v}^{S}\left(L_{i}^{S}\right)}{F_{v}^{S}\left(L_{i+1}^{S}\right)-F_{v}^{S}\left(L_{i}^{S}\right)}=\frac{F_{v}^{P}\left(L_{e}^{P}\right)-F_{v}^{P}\left(L_{i}^{P}\right)}{F_{v}^{P}\left(L_{i+1}^{P}\right)-F_{v}^{P}\left(L_{i}^{P}\right)} .
$$

It is easy to deduce that

$$
F_{v}^{P}\left(L_{e}^{P}\right)=F_{v}^{P}\left(L_{i}^{P}\right)+\frac{\left(F_{v}^{S}\left(L_{e}^{S}\right)-F_{v}^{S}\left(L_{i}^{S}\right)\right) *\left(F_{v}^{P}\left(L_{i+1}^{P}\right)-F_{v}^{P}\left(L_{i}^{P}\right)\right)}{F_{v}^{S}\left(L_{i+1}^{S}\right)-F_{v}^{S}\left(L_{i}^{S}\right)} .
$$

Then, the corresponding location in prone is

$$
L_{e}^{P}=\left(F_{v}^{P}\right)^{-1}\left(F_{v}^{P}\left(L_{e}^{P}\right)\right),
$$

where $\left(F_{v}^{P}\right)^{-1}$ is a mapping to find the locations according to the $F_{v}^{P}$.

\section{Experiments and Results}

\subsection{Data Acquisition and Pre-processing}

Each patient was limited to the low-fibre diet beginning 1 day before the scheduled morning CTC. Colonic catharsis was achieved with mannitol on the evening before the examination. Note that, this preparation procedure is close to the prepless VC procedure and is pleasantly accepted by recruited patients. Before the examination, the colon was distended with $1500 \mathrm{~mL}$ of water-soluble iodinated contrast medium using a manual insufflators with a small rectal catheter. Examinations were performed in supine positions on a 128-MDCT scanner (SOMATOM Definition AS, SIEMENS, Germany). CT technique consisted of 5.00mm collimation, 1.375 : 1 pitch, $1-\mathrm{mm}$ reconstruction interval, $120 \mathrm{kVp}$, and 50-100 mAs. Twenty cases are used for the experiment and all cases were of diagnostic quality, contain mainly fluid, but could contain feces as well. 10 patients (50\%) had 20 confirmed polyps: 5 polyps were $5-10 \mathrm{~mm}, 12$ polyps were $3-4 \mathrm{~mm}$ and 3 polyp was under $3 \mathrm{~mm}$. 10 patients (50\%) were normal.

In the pre-processing step, the colonic lumens are extracted from the CTC datasets by use of a level-set segmentation method [11. Actually, any other colon segmentation technique could potentially be used for the pre-processing step. Colon lumen surface is extracted after the segmentation.

\subsection{Experimental Results}

Algorithms described in Sec. 3.1 and Sec. 2.2 are performed to process all the datasets. In order to evaluate the performance of the registration we proposed, a doctor experienced in the interpretation of CTC cases evaluated the $2 D$ and $3 D$ visualizations of the colon by use of our software tool plugged into the $\mathrm{VC}$ software plateform by Yulonn Medical Imaging Company (Fig. 2). Our software 


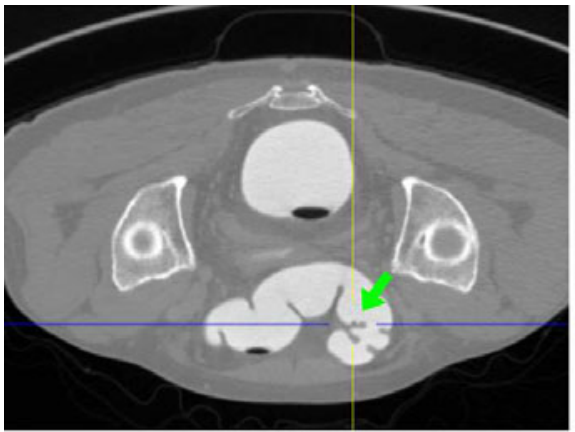

(a)

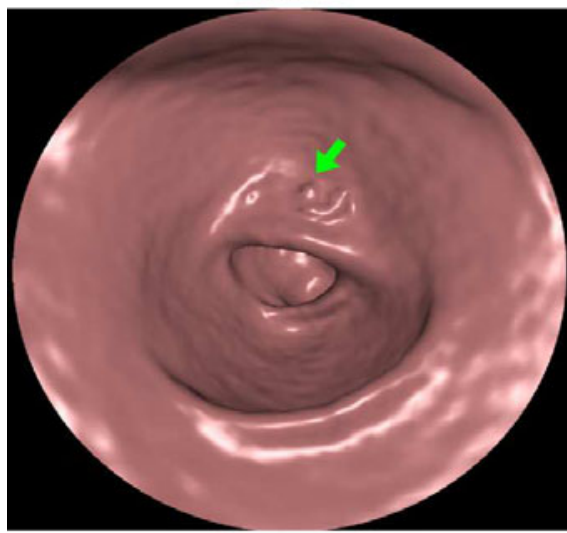

(c)

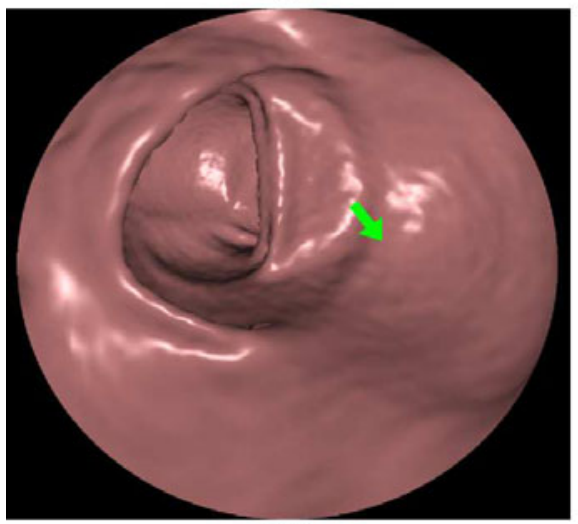

(e)

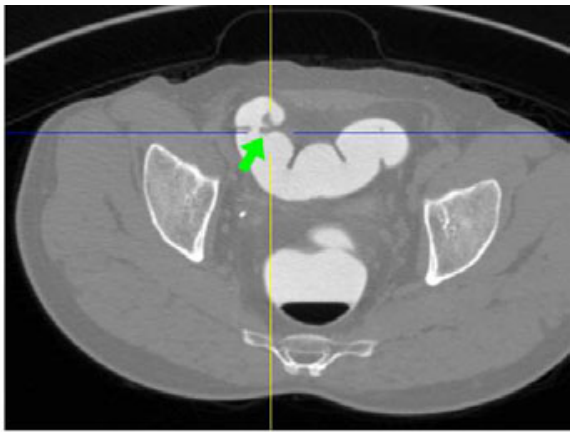

(b)

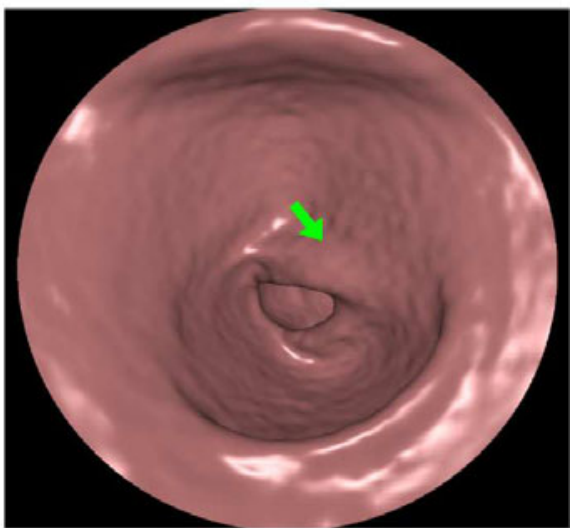

(d)

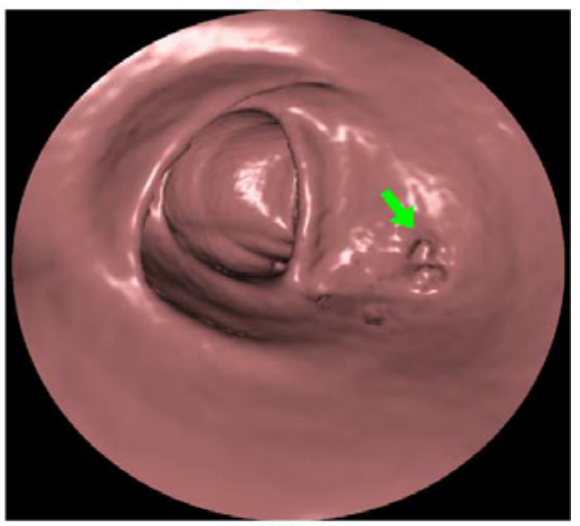

(f)

Fig. 2. Illustration of false positive reduction. In (a), in supine position it has a false positive which the green arrow pointing at. In (b), in prone position it confirms that it is a pseudo-polyp but not a true polyp. (c) and (d) is the virtual colonoscopy of (a) and (b). (e) and (f) show another example which has false positives in prone position and can be confirmed as pseudo-polyps in supine position. 
Table 1. Results of comparison between shape spectrum method and deformable model

\begin{tabular}{|l|c|}
\hline Method & Accuracy $(\mathrm{mm})$ \\
\hline \hline Shape Spectrum Method & $12.6 \pm 4.20$ \\
Deformable Model & $15.4 \pm 6.30$ \\
\hline
\end{tabular}

tool and algorithm are implemented with $\mathrm{C}++$. The tool displays the $3 D \mathrm{VC}$ of two intra-patient colon scans: supine position on the left and prone position on the right. By clicking on either $3 D$ colon lumen, it finds the correspondence region in the other colon lumen, as well as the views of $2 D$ CTC updated simultaneously to show the corresponding location in both colon scans. A doctor can check any location he feels interested in and our system automatically updates the corresponding regions in an opposite position. The application of the method resulted in a registration accuracy of $12.6 \pm 4.20 \mathrm{~mm}$ over 20 datasets. We compared our method with the deformable model method 6] based on our datasets. The comparison result is shown in Table. 1] Our method outperforms the deformable model approach, and more importantly, our method does not create any artifacts resulting in pseudo-polyps but the deformable model would.

We integrate our method to the VC software for FP reduction. First of all, the polyp candidates are selected by asking doctors to go through all the datasets to find any polyp-like protrusions. 78 polyp candidates from all the CTC datasets are presented, among which 20 polyps are true positives and 58 polyps are false positives. Since it is impossible for any registration algorithm to find exact pointto-point correspondence, in practices, it is common to set a interval, $F_{v}$, where the correspondent locations fall in. Specifically, the correspondent interval locations Interval ${ }^{P}\left(L_{e}^{S}\right)$ in prone which are the correspondent locations to $L_{e}^{S}$ in supine would be

$$
\text { Interval }^{P}\left(L_{e}^{S}\right)=\left[\left(F_{v}^{P}\right)^{-1}\left(F_{v}^{P}\left(L_{e}^{P}\right)-\varepsilon\right),\left(F_{v}^{P}\right)^{-1}\left(F_{v}^{P}\left(L_{e}^{P}\right)+\varepsilon\right)\right] .
$$

Then we check the polyps candidates in supine position and the correspondent interval locations in prone; and check the polyps candidates in prone position and the correspondent interval location in supine as well. Fig. 2 shows examples of FP reduction. By checking the correspondence, 48 FPs are spotted out, which is $83 \%$ reduction compared with independent processing of the datasets, with no true-positive is eliminated.

\section{Conclusion}

We have developed a novel automatic method for registration of the supine and prone CTC datasets. The application of the method resulted in an accuracy of $12.6 \pm 4.20 \mathrm{~mm}$ over 20 datasets with the number of false positive reduced by $83 \%$ compared with independent processing of the datasets. The experimental results indicate that the method is useful in improving the specificity of the polyps in CTC. In the feature work, we will combine with advanced classification methods for further false positive reduction. 


\section{Acknowledgements}

This work is supported in part by the National Science Foundation research grants awarded to Dr. J. Hua, including NSF IIS-0915933, NSF IIS-0937586 and NSF IIS-0713315. We would also like to thank Yulonn Medical Imaging Co., Ltd. for providing us the data and the Virtual Colonoscopy software platform.

\section{References}

1. Vining, D.J.: Virtual colonoscopy. Gastrointestinal Endosc. Clin. N. Am. 7(2), 285291 (1997)

2. Zou, G., Hua, J., Muzik, O.: Non-rigid Surface Registration Using Spherical Thinplate Splines. In: Ayache, N., Ourselin, S., Maeder, A. (eds.) MICCAI 2007, Part I. LNCS, vol. 4791, pp. 367-374. Springer, Heidelberg (2007)

3. Duan, Y., Hua, J., Qin, H.: Interactive Shape Modeling Using Lagrangian Surface Flow. The Visual Compute 21(5), 279-288 (2005)

4. Li, P., Napel, S., Acar, B., Paik, D.S., Jeffrey, R.B., Beaulieu, C.F.: Registration of central paths and colonic polyps between supine and prone scans in computed tomography colonography: Pilot study. Med. Phys. 31(10), 2912-2923 (2004)

5. Nain, D., Haker, S., Grimson Jr., W.E.L., Wells, W.M., Ji, H., Kikinis, R., Westin, C.-F.: Intra-patient Prone to Supine Colon Registration for Synchronized Colonoscopy. In: Dohi, T., Kikinis, R. (eds.) MICCAI 2002. LNCS, vol. 2489, pp. 573-580. Springer, Heidelberg (2002)

6. Suh, W.J., Wyatt, C.L.: Deformable registration of supine and prone colons using cneterline analysis. In: 4th IEEE International Symposium on Biomedical Imaging, pp. 708-711 (2007)

7. Näppi, J., Okamura, A., Frimmel, H., Dachman, A., Yoshida, H.: Region-based Supine-prone Correspondence for the Reduction of False-positive CAD Polyp Candidates in CT Colonography. Academic Radiology 12(6), 695-707 (2005)

8. Rustamov, R.M.: Laplace-Beltrami eigenfunctions for deformation invariant shape representation. In: Symposium on Geometry Processing, pp. 225-233 (2007)

9. Reuter, M., Wolter, F.E., Peinecke, N.: Laplace-Beltrami spectra as Shape-DNA of surfaces and solids. Comput. Aided Des. 38(4), 342-366 (2006)

10. Lévy, B.: Laplace-Beltrami eigenfunctions: towards an algorithm that understands geometry. In: IEEE International Conference on Shape Modeling and Applications (2006)

11. Lankton, S., Tannenbaum, A.: Localizing region-based active contours. IEEE Transactions on Image Processing 17(11), 2029-2039 (2008) 